\title{
Características seminales de verracos alojados en ambiente controlado ubicado en trópico cálido
}

\author{
Seminal characteristics of boars housed in a controlled environment located \\ in the warm tropics
}

\author{
Derwin Calatayud-Márquez ${ }^{1,3}$, Armando Quintero-Moreno
}

\section{Resumen}

\begin{abstract}
El estudio tuvo como objetivo evaluar las características seminales de verracos alojados en un sistema de ambiente controlado en bosque seco tropical en una granja porcina comercial ubicada en el estado Zulia, Venezuela. Se analizaron 6154 evaluaciones seminales (volumen, espermatozoides totales, concentración, motilidad y número de dosis por eyaculado) de 95 verracos de la línea comercial Topigs ${ }^{\circledR}$, desde septiembre de 2006 hasta julio de 2010. Los verracos se encontraban alojados en un sistema de ambiente controlado de confort higrotérmico (Chore.Time. 2529,676; modelo 8-B1.5). Se observaron valores promedios de $253.2 \pm 57.0 \mathrm{ml}$ para volumen seminal, $3.59 \pm 0.2 \mathrm{de}$ motilidad individual progresiva, $322.1 \times 10^{6} \pm 98.2$ espermatozoides $/ \mathrm{ml}$ para la concentración espermática, $81.2 \times 10^{9} \pm 26.1$ espermatozoides totales por eyaculado y una producción de $27.0 \pm 8.7$ dosis seminales por cada eyaculado. Se obtuvo mejor producción seminal en enero y febrero y menores valores en noviembre y diciembre. Existe un efecto altamente significativo $(p<0.001)$ del mes sobre todas las características seminales con excepción del volumen ( $\mathrm{p}>0.05)$. El sistema de alojamiento de ambiente controlado no evita la notable variación de eyaculados dentro y entre los meses evaluados.
\end{abstract}

Palabras clave: alojamiento, cerdo, clima tropical, estaciones del año, semen

\footnotetext{
${ }^{1}$ Universidad de Ciencias Aplicadas y Ambientales, Facultad de Ciencias Agropecuarias. Grupo de Investigación Ciencia Animal, Bogotá Colombia

${ }^{2}$ Universidad del Zulia, Facultad de Ciencias Veterinarias, Laboratorio de Andrología, Maracaibo, Venezuela

${ }^{3}$ E-mail: dcalatayud@udca.edu.co
}

Recibido: 14 de noviembre de 2020

Aceptado para publicación: 18 de mayo de 2021

Publicado: 24 de agosto de 2021

CLos autores. Este artículo es publicado por la Rev Inv Vet Perú de la Facultad de Medicina Veterinaria, Universidad Nacional Mayor de San Marcos. Este es un artículo de acceso abierto, distribuido bajo los términos de la licencia Creative Commons Atribución 4.0 Internacional (CC BY 4.0) [https:// creativecommons.org/licenses/by/4.0/deed.es] que permite el uso, distribución y reproducción en cualquier medio, siempre que la obra original sea debidamente citada de su fuente original 
The aim of this study was to evaluate the seminal characteristics of boars housed in a controlled environment system in tropical dry forest in a commercial pig farm located in the state of Zulia, Venezuela. In total, 6154 seminal evaluations (volume, total sperm, concentration, motility and number of doses per ejaculate) of 95 boars from the Topigs ${ }^{\circledR}$ commercial line were analysed from September 2006 to July 2010. The boars were housed in a controlled environmental system of hygrothermal comfort (Chore.Time. 2529,676; model 8-B1.5). Average values of $253.2 \pm 57.0 \mathrm{ml}$ were observed for seminal volume, 3.59 \pm 0.2 for progressive individual motility, $322.1 \times 10^{6} \pm 98.2 \mathrm{spermatozoa} / \mathrm{ml}$ for sperm concentration, $81.2 \times 10^{9} \pm 26.1$ total spermatozoa per ejaculate and a production of $27.0 \pm$ 8.7 semen doses for ejaculate. Better seminal production was obtained in January and February and lower values in November and December. There is a highly significant effect $(p<0.001)$ of the month on all seminal characteristics except for volume $(p>0.05)$. The controlled environment housing system does not prevent the notable variation of ejaculates within and between the months evaluated.

Key words: housing, pigs, tropical climate, seasons, semen

\section{INTRODUCCIÓN}

En la reproducción de la especie porcina existen puntos críticos, como la fertilidad del verraco (Savic et al., 2013), la cual ejerce un alto impacto sobre la eficiencia reproductiva del rebaño. Por tal motivo, el éxito de la técnica de inseminación artificial (IA) implica la identificación de verracos con calidad óptima del semen y con capacidad de adaptarse a los cambios climáticos. En países de clima templado, la eficiencia reproductiva depende en parte del efecto de las variaciones estacionales que enfrentan los verracos (Fuentes et al., 1992; Wisokinska et al., 2009; Savic et al., 2013), además de factores adicionales que influyen en la producción seminal, tales como la raza (Knecht et al., 2014; Caisin y Snitco, 2016; Žaja et al., 2016), nutrición (Murphy et al., 2017), edad (Huang et al., 2016; Banaszewska y Kondracki, 2012), socialización (Dysart, 2015), así como el desarrollo testicular (Ford y Wise, 2010) y los cambios histomorfológicos del tejido testicular (Mažeika et al., 2012).
En los países tropicales, de otra parte, no se tiene grandes variaciones con respecto a la duración de luz durante el día, ni grandes fluctuaciones de temperatura ambiental (TA) durante el año. No obstante, se documenta una disminución del rendimiento reproductivo en algunas épocas del año (Suriyasomboon et al., 2004; Peña et al., 2019), producto del estrés calórico (EC) extendido durante varios meses. En este sentido, el EC es uno de los principales factores a tomar en cuenta en el porcino, debido a que posee pocas glándulas sudoríparas en comparación con otras especies que, utilizan la sudoración como mecanismo de disipación calórica, situación que obliga al cerdo a disipar calor mediante el refrescamiento evaporativo (Bracke y Spoolder, 2011).

La capacidad adaptativa o sensibilidad individual de los verracos a factores estacionales puede generar variaciones importantes en los eyaculados (Wisokinska et al., 2009). De hecho, la eficiencia reproductiva se ve afectada durante y después de la temporada de calor, ya que el EC 
genera una producción excesiva de cortisol, lo cual afecta la producción espermática $\mathrm{y}$, por ende, la calidad seminal (Olczak et al., 2015). No obstante, el rendimiento reproductivo del porcino se puede mejorar al manejar eficientemente las condiciones ambientales. Por tal motivo, algunas granjas han incorporado mecanismos de ambiente controlado (AC) de refrigeración por evaporación para manejar y disminuir la excesiva TA y humedad relativa (HR) del ambiente que se observa en zonas tropicales, reduciendo así el EC (Suriyasomboon et al., 2004), mejorando la sensación térmica e incrementando el consumo de alimento.

En la actualidad existe poca información del rendimiento de porcinos alojados en AC bajo condiciones de trópico cálido, hecho que motivó a realizar esta investigación para caracterizar los parámetros seminales convencionales de porcinos alojados en un sistema de $\mathrm{AC}$ en trópico cálido con TA anual que sobrepasa los $30^{\circ} \mathrm{C}$ en los meses más cálidos.

\section{Materiales y Métodos}

\section{Ubicación Geográfica}

La evaluación se realizó en un sistema de producción de cerdos (PROPORCA C.A. ${ }^{\circledR}$ ) ubicado en el municipio San Francisco del Estado Zulia, Venezuela, en una zona caracterizada agroecológicamente como bosque seco tropical cálido con temperatura ambiental, humedad relativa e índice temperatura humedad (ITH) descritos en el Cuadro 1. Los datos climáticos fueron registrados quincenalmente y fueron obtenidos de la estación meteorológica más cercana.

\section{Unidades Experimentales}

Se evaluaron 6154 eyaculados provenientes de 95 verracos de la línea comercial Topigs $^{\circledR}$, con edades comprendidas entre 12 y 36 meses y pesos corporales entre los 180 y $300 \mathrm{~kg}$ entre septiembre de 2006 y julio de 2010. Los verracos fueron mantenidos en un área bajo $\mathrm{AC}$ de confort higrotérmico (Chore.Time. 2529,676; modelo 8-B1.5); con una dieta diaria (una vez/día) basada en alimento concentrado de exclusividad para verracos (PROPORCA C.A. ${ }^{\circledR}$ ), buena disponibilidad de agua, además de un programa sanitario riguroso y estrictas normas de bioseguridad.

\section{Extracción, Colección y Procesamiento del Semen}

La extracción se realizó una vez por semana a cada verraco entre las 07:00 y 11:00 $\mathrm{h}$, mediante la técnica de mano enguantada. El eyaculado se colectó en un recipiente plástico de $500 \mathrm{ml}$ con filtro dentro de un termo temperado a $37^{\circ} \mathrm{C}$ y debidamente protegido. Previo a la colecta se realizó la eliminación de restos de orina y fluidos de la bolsa prepucial del verraco, acto seguido se obtuvo la fracción rica en espermatozoides. Se trasladó la muestra al laboratorio y se colocó en baño María a $37^{\circ} \mathrm{C}$ para valorar la calidad seminal y procesar las nuestras idóneas. Solo fueron estudiadas las muestras seminales con volumen (V), color, olor y aspecto de acuerdo con los patrones establecidos en la granja: motilidad individual progresiva $(\mathrm{MI}) \geq 3$, número total de espermios por eyaculado (ET) $>24 \times 10^{9}$. Del mismo modo, se descartaron muestras con color y/o apariencia anormal (rojizos, pardos, grises, amarillentos o con presencia de flósculos de pus), o aglutinaciones que denoten contaminación de la muestra seminal.

\section{Características Seminales}

El volumen fue determinado en una balanza electrónica, realizando la conversión en mililitros. Se evaluó el color, olor y aspecto del eyaculado. La MI y la concentración (CE) se valoraron con un microscopio óptico $\left(\mathrm{Nikon}^{\circledR}\right)$. La CE se valoró utilizando una cámara de Neubauer ${ }^{\circledR}$ a 40X; mientras que la MI se determinó con aumento de 10X. La 
Cuadro 1. Distribución mensual de la temperatura ambiental (TA), humedad relativa (HR) e índice temperatura humedad (ITH) en el área geográfica bajo estudio durante los años 2006-2010

\begin{tabular}{|c|c|c|c|c|c|}
\hline \multirow{3}{*}{ Mes } & \multicolumn{5}{|c|}{ Parámetros de medio ambiente } \\
\hline & \multicolumn{3}{|c|}{ Temperatura $\left({ }^{\circ} \mathrm{C}\right)$} & \multirow{2}{*}{$\begin{array}{l}\text { Humedad } \\
\text { relativa }(\Phi)\end{array}$} & \multirow{2}{*}{$\mathrm{ITH}^{*}$} \\
\hline & Media & Máxima & Mínima & & \\
\hline Enero & 28.50 & 32.69 & 23.26 & 68.10 & 78.84 \\
\hline Febrero & 29.01 & 33.42 & 23.26 & 64.86 & 79.14 \\
\hline Marzo & 29.06 & 33.11 & 24.51 & 67.52 & 79.62 \\
\hline Abril & 29.71 & 33.55 & 25.15 & 69.33 & 80.81 \\
\hline Mayo & 29.99 & 33.68 & 25.41 & 72.39 & 81.68 \\
\hline Junio & 29.98 & 33.88 & 25.43 & 89.52 & 84.33 \\
\hline Julio & 30.24 & 34.08 & 25.37 & 70.72 & 81.84 \\
\hline Agosto & 30.32 & 33.92 & 25.33 & 76.53 & 82.84 \\
\hline Septiembre & 30.43 & 34.17 & 25.24 & 77.37 & 83.13 \\
\hline Octubre & 29.33 & 32.73 & 24.75 & 81.52 & 82.05 \\
\hline Noviembre & 29.32 & 32.63 & 24.96 & 80.74 & 81.86 \\
\hline Diciembre & 28.93 & 32.87 & 23.59 & 74.60 & 80.47 \\
\hline
\end{tabular}

${ }^{*} \mathrm{ITH}=0.8^{\circ} \mathrm{Ta}+(($ humedad relativa del aire/100)*(Ta -14.3$))+46.4$ (Thom EC, 1959. The discomfort index. Weatherwise 12:57-59)

calidad del movimiento de los espermatozoides se realizó bajo una escala de 0 a 5 , en el cual: $0=$ No se observaron movimientos; 1 = Escaso movimiento, únicamente la cola tiembla ligeramente; 2 = Espermatozoides con desplazamientos en círculos y algunos progresivos; 3 = Movimientos progresivos y sinuosos; $4=$ Movimiento progresivo rápido y $5=$ Movimientos progresivos muy rápidos. El cálculo de ET y Número de dosis por eyaculado (NDE) se calcularon con fórmulas convencionales.

El eyaculado que obtuvo el mínimo de requerimientos establecidos para su uso en IA se procesó con un diluyente comercial $\left(\mathrm{MRA}^{\mathbb{}}\right)$ y se envasó en botellas plásticas con capacidad para $90 \mathrm{ml}$ con una CE de $4 \times 10^{9}$ espermatozoides por dosis. Las dosis obtenidas se colocaron a temperatura de laboratorio $\left(22-25^{\circ} \mathrm{C}\right)$ durante $2 \mathrm{~h}$ y cubiertas para evitar el exceso de luz. Luego se llevaron a un refrigerador a temperatura de $15-18{ }^{\circ} \mathrm{C}$. El semen fue utilizado a partir de las 18 horas de almacenamiento. La IA se realizó con la técnica tradicional (3 IA/cerda) obteniendo un $90.1 \%$ de fertilidad durante el período de evaluación (Cuadro 2).

\section{Análisis Estadístico}

Los datos se introdujeron en el programa Microsoft Excel 2008 para Windows, v. 12.0, para luego ser exportados al programa Statistical Analysis System para Windows, v. 9.1.3 (SAS Institute) y ser procesados me- 
Cuadro 2. Porcentaje (\%) de fertilidad en el rebaño porcino obtenido en la granja durante el periodo 2006-2010

\begin{tabular}{lc}
\hline Mes & $\begin{array}{c}\text { Fertilidad } \\
(\%)\end{array}$ \\
\hline Enero & 91.3 \\
Febrero & 89.7 \\
Marzo & 91.6 \\
Abril & 90.3 \\
Mayo & 89.1 \\
Junio & 87.9 \\
Julio & 91.4 \\
Agosto & 91.7 \\
Septiembre & 89.1 \\
Octubre & 87.6 \\
Noviembre & 90.7 \\
Diciembre & 90.6 \\
\hline
\end{tabular}

diante el Modelo Lineal General (PROC GLM). El mes del año (enero-diciembre) fue evaluado como variable discreta independiente y las variables de calidad seminal: V, MI, CE, ET y NDE) como variables cuantitativas dependiente del efecto mes del año. Las variables línea genética (maternal y/o terminal de Topigs ${ }^{\circledR}$ ) y año de evaluación (20062010) se utilizaron como variables discretas independientes para ajustar el modelo estadístico. Cuando se detectaron diferencias significativas, se cuantificaron mediante la prueba de medias mínimas cuadráticas (LSMEANS).

\section{Resultados}

En el Cuadro 3 se presentan los promedios del mes del año sobre MI, CE, ET, y NDE con diferencias altamente significativas entre meses $(p<0.01)$, con excepción del mes del año sobre el $\mathrm{V}$ del eyaculado $(\mathrm{p}=0.2511)$.
Las características seminales distribuidas por mes del año se pueden observar en el Cuadro 4. No hubo significancia estadística sobre el V, aunque se observó una propensión numérica mayor en los primeros meses del año, especialmente en marzo (283.4 $\mathrm{ml})$. No se observó variación entre los valores de MI en la mayoría de los meses evaluados (enero a junio y septiembre-noviembre); sin embargo, se encontró diferencia significativa $(\mathrm{p}<0.0001)$ de estos meses con los valores obtenidos en julio y diciembre, donde se obtuvieron valores inferiores.

Los parámetros CE, ET y NDE presentan rendimientos similares durante todo en el año. No obstante, durante el mes de enero se observaron los mejores valores $\left(331.2 \times 10^{6}\right.$; $94.5 \times 10^{9}$ y 31.5 , respectivamente), siendo además significativamente superiores $(\mathrm{p}<0.05)$ a los valores observados en los demás meses. Por otro lado, se distingue una disminución progresiva e importante en los valores promedios para estas características a partir del segundo trimestre del año, para finalmente alcanzar niveles mínimos de $72.8 \times 10^{9}$ y $69.3 \times 10^{9}$ para ET, concentraciones de $251.4 \times 10^{6}$ y $239.2 \times 10^{6} / \mathrm{ml}$, así como NDE de 24.2 y 23.1 en noviembre y diciembre, respectivamente. Así mismo, se puede notar el amplio rango de variación con respecto para estas características dentro y entre los meses evaluados.

\section{Discusión}

En general, se observaron promedios superiores para volumen, espermatozoides totales e inferiores para concentración y motilidad con respecto a lo reportado por Suriyasomboon et al. (2004) en verracos alojados en ambiente controlado en Tailandia y por Fuentes et al. (1992) en ambiente convencional de trópico venezolano. Además, se pudo observar un amplio rango de variaciones de los eyaculados, lo cual concuerda con lo evidenciado por Wisokiñska et al. (2009), 
Cuadro 3. Efecto del mes del año sobre características seminales y número de dosis por eyaculado de verracos alojados en sistema de ambiente controlado en trópico cálido

\begin{tabular}{lcc}
\hline Característica seminal & Media & Valor de P \\
\hline Volumen $(\mathrm{ml})$ & $253.2 \pm 57.0$ & 0.2511 \\
Concentración $\left(\mathrm{x} 10^{6} / \mathrm{ml}\right)$ & $322.1 \pm 98.2$ & $<0.0001$ \\
Espermatozoides totales $\left(\mathrm{x} 10^{9}\right)$ & $81.2 \pm 26.1$ & $<0.0001$ \\
Motilidad & $3.5 \pm 0.2$ & 0.0002 \\
Número de dosis & $27.0 \pm 8.7$ & $<0.0001$ \\
\hline
\end{tabular}

Cuadro 4. Características seminales en porcinos alojados en ambiente controlado en trópico cálido según el mes del año

\begin{tabular}{lccccc}
\hline \multirow{2}{*}{ Mes } & \multicolumn{5}{c}{ Parámetros convencionales de evaluación seminal } \\
\cline { 2 - 6 } & $\mathrm{V}(\mathrm{ml})$ & $\mathrm{MI}\left(\mathrm{N}^{\mathrm{o}}\right)$ & $\mathrm{CE}\left(\mathrm{x} 10^{6}\right)$ & $\mathrm{ET}\left(\mathrm{x} 10^{\mathrm{9}}\right)$ & $\mathrm{NDE}\left(\mathrm{N}^{\mathrm{o}}\right)$ \\
\hline Enero & $282.6 \pm 1.5$ & $3.63 \pm 0.01^{\mathrm{a}}$ & $331.2 \pm 3.4^{\mathrm{a}}$ & $94.5 \pm 0.1^{\mathrm{a}}$ & $31.5 \pm 0.05^{\mathrm{a}}$ \\
Febrero & $281.9 \pm 1.7$ & $3.58 \pm 0.01^{\mathrm{a}}$ & $324.6 \pm 3.9^{\mathrm{ab}}$ & $92.1 \pm 0.1^{\mathrm{b}}$ & $30.7 \pm 0.05^{\mathrm{b}}$ \\
Marzo & $283.4 \pm 1.5$ & $3.60 \pm 0.01^{\mathrm{a}}$ & $314.5 \pm 3.5^{\mathrm{bc}}$ & $89.7 \pm 0.1^{\mathrm{c}}$ & $29.9 \pm 0.05^{\mathrm{c}}$ \\
Abril & $281.4 \pm 1.5$ & $3.57 \pm 0.01^{\mathrm{a}}$ & $305.2 \pm 3.5^{\mathrm{cd}}$ & $87.2 \pm 0.1^{\mathrm{d}}$ & $29.0 \pm 0.05^{\mathrm{d}}$ \\
Mayo & $281.7 \pm 1.4$ & $3.59 \pm 0.01^{\mathrm{a}}$ & $295.2 \pm 3.2^{\mathrm{de}}$ & $84.7 \pm 0.1^{\mathrm{e}}$ & $28.2 \pm 0.04^{\mathrm{e}}$ \\
Junio & $280.2 \pm 1.4$ & $3.61 \pm 0.01^{\mathrm{a}}$ & $289.4 \pm 3.2^{\mathrm{ef}}$ & $83.1 \pm 0.1^{\mathrm{f}}$ & $27.7 \pm 0.04^{\mathrm{f}}$ \\
Julio & $280.4 \pm 1.5$ & $3.54 \pm 0.01^{\mathrm{b}}$ & $283.4 \pm 3.5^{\mathrm{ef}}$ & $81.4 \pm 0.1^{\mathrm{g}}$ & $27.1 \pm 0.05^{\mathrm{g}}$ \\
Agosto & $280.7 \pm 1.7$ & $3.58 \pm 0.01^{\mathrm{a}}$ & $274.9 \pm 3.8^{\mathrm{fg}}$ & $79.4 \pm 0.1^{\mathrm{h}}$ & $26.4 \pm 0.05^{\mathrm{h}}$ \\
Septiembre & $278.6 \pm 1.4$ & $3.57 \pm 0.01^{\mathrm{a}}$ & $278.9 \pm 3.2^{\mathrm{efg}}$ & $79.7 \pm 0.1^{\mathrm{h}}$ & $26.5 \pm 0.04^{\mathrm{h}}$ \\
Octubre & $278.5 \pm 1.3$ & $3.57 \pm 0.01^{\mathrm{a}}$ & $266.8 \pm 2.9^{\mathrm{gh}}$ & $77.5 \pm 0.1^{\mathrm{i}}$ & $25.8 \pm 0.04^{\mathrm{i}}$ \\
Noviembre & $278.2 \pm 1.3$ & $3.55 \pm 0.01^{\mathrm{a}}$ & $251.4 \pm 2.9^{\mathrm{i}}$ & $72.8 \pm 0.1^{\mathrm{j}}$ & $24.2 \pm 0.04^{\mathrm{j}}$ \\
Diciembre & $278.6 \pm 1.3$ & $3.52 \pm 0.01^{\mathrm{b}}$ & $239.2 \pm 2.9^{\mathrm{i}}$ & $69.3 \pm 0.1^{\mathrm{k}}$ & $23.1 \pm 0.04^{\mathrm{k}}$ \\
\hline V
\end{tabular}

V: volumen; MI: motilidad individual; CE: concentración espermática; ET: espermatozoides totales en el eyaculado; NDE: número de dosis obtenida por cada eyaculado Letras diferentes $(a-b, a-i, a-k)$ en la misma columna significa diferencias estadísticas $(p<0.01)$ entre meses

indicando una respuesta adaptativa o de sensibilidad individual de los verracos a determinados ambientes o factores estacionales que pueden generar variaciones importantes en los eyaculados.
Ahora bien, aunque algunas áreas geográficas tropicales poseen menos variación climática que en otras áreas del mundo, el trópico se caracteriza por poseer diversos microclimas con características diversas en- 
tre cada uno de ellos. No obstante, cada microclima posee características agro-climatológicas constantes a lo largo del año, hecho evidenciable en el Cuadro 1 para humedad y temperatura. En este orden de ideas, Ewel et al. (1976) ubican la granja porcina evaluada en una zona de vida caracterizada como bosque seco tropical con presencia de temperaturas altas a lo largo del año, siendo extremas $\left(35\right.$ a $\left.40{ }^{\circ} \mathrm{C}\right)$ en ciertas horas del día (10:00 a 16:00) y en algunos meses del año (junio-septiembre).

En este sentido, los valores promedios de las características seminales reportados en esta investigación, exceptuando el volumen, podrían ser explicados por el efecto de alta temperatura y humedad relativa ambiental que afectarían el microclima tanto en los sistemas de alojamiento convencional como en los sistemas de enfriamiento por evaporación de forma controlada; este último descrito en el presente estudio y que concuerda con los reportes en clima tropical en relación a las consecuencias detrimentales de las características seminales de los verracos a pesar de estar en ambientes controlados (Suriyasomboon et al., 2004). Esta situación deriva del hecho que este tipo de ambiente controlado no es capaz de propiciar temperaturas inferiores a $\operatorname{los} 28^{\circ} \mathrm{C}$ cuando la temperatura del medio ambiente supera $\operatorname{los} 35^{\circ} \mathrm{C}$ y humedad relativa elevada. Sin embargo, como esta situación solo ocurre en algunas horas del día, el estrés calórico ocasionado a los reproductores no es permanente, siendo su rendimiento reproductivo adecuado, incluso en los meses de mayor índice humedadtemperatura ambiental.

Otro punto impórtate a destacar, para el mejor entendimiento de los resultados del presente estudio, hace referencia a que, aunque la domesticación puede haber conducido a la pérdida parcial o total de la capacidad de adaptativa a los cambios estacionales en las cerdas y verracos, estos sin embargo, todavía muestran características típicas de su antecesor silvestre en lo referente a mayor actividad de apareamiento durante el periodo de otoño e invierno (Wisokiñska et al., 2009). Bajo esta perspectiva, existe la posibilidad de que el efecto de la estación sea principalmente la consecuencia de las diferencias en el fotoperiodo natural y que la calidad del semen sea mayoritariamente afectada en la época de otoño (Petrocelli et al., 2015). En este orden, otras investigaciones también han demostrado que el fotoperiodo influye en la calidad seminal de los verracos (Yeste et al., 2010; Knecht et al., 2013; Saviæ y Petroviæ, 2015). Sin embargo, Gómez (2016) obtuvo menor volumen en el fotoperiodo corto de invierno, pero con incremento de la concentración de manera compensatoria, obteniendo un número uniforme de espermatozoides totales durante todo el año.

Los resultados del presente estudio son en parte similares a otras investigaciones, dado que otros autores confirmaron los efectos de la época en la variabilidad de las características de los eyaculados analizados (Kunowskaslósarz y Makowska, 2011; Pinart y Puigmulé, 2013; Koprianiuk et al., 2014; Zasiadczyk et al., 2015; Fraser et al., 2016). No obstante, estos cambios no son iguales en las diferentes regiones evaluadas (Argenti et al., 2018).

Con respecto al volumen seminal, aunque no se observó efecto del mes sobre esta característica, la concordancia observada con respecto a los demás estudios se deba, posiblemente, a una rápida capacidad adaptativa del cerdo para esta característica (Rivera et al., 2005), y al estar expuesto a menores variaciones diurnas de temperatura ofrecidas por el sistema de alojamiento controlado. De este modo, los valores más altos se obtuvieron entre enero y marzo y los menores valores desde septiembre a diciembre. Este hecho es similar a lo reportado por Fuentes et al. (1992) en Venezuela en ambiente convencional donde el mayor volumen seminal se observó en el primer trimestre; sin embrago, no coincide con los valores de los demás meses. 
Por otra parte, la motilidad, es considerada como uno de los parámetros más importantes a medir en los espermatozoides (Kunowska-slósarz y Makowska, 2011), y que en conjunto con la morfología son indicadores sensibles del estrés por calor (Lipenský et al., 2010). En este sentido, se coincide con Fuentes et al. (1992) y con Savic et al. (2013), los cuales demuestran el efecto de la época sobre la motilidad espermática. La concentración siguió un patrón similar a las otras variables estudiadas, con picos superiores en enero y febrero, lo cual contrasta con Fuentes et al. (1992), donde la concentración fue mayor entre mayo y junio. Asimismo, difiere de lo reportado por Peña et al. (2019) en Australia, en el cual el verano tropical (temporada alta de lluvias y calor) entre noviembre y abril, reduce la concentración, además de daños en el ADN de los espermatozoides.

La literatura científica coincide en que la época o, en este caso, el mes del año influye sobre la mayoría de las características seminales; sin embargo, es importante destacar que el clima varía según la localización geográfica (Argenti et al., 2018). Temperaturas más elevadas en determinados momentos del año pueden influir negativamente en el proceso de espermatogénesis en cerdos (Malmgren, 1989), lo cual se ha descrito de manera similar en ratones, con la consecuente disminución de células germinales en los testículos, debido a apoptosis y desregularización de complejos de unión de las células de sertoli (Cai et al., 2011; Li et al., 2013). Así mismo, las diferencias relacionadas con la estacionalidad se ven asociadas al rendimiento metabólico de espermatozoides y actividad de enzimas antioxidantes del tracto reproductivo del verraco (Dziekoñska et al., 2014). Flowers (2015) reporta que la mayoría de los aspectos medioambientales a los que están expuestos los verracos tienen capacidad para influir en la producción y calidad espermática.

\section{Conclusiones}

- Existe efecto significativo $(\mathrm{p}<0.05)$ del mes sobre todas las características seminales con excepción del volumen, siendo la mejor producción seminal para el área geográfica evaluada en los meses de enero y febrero.

- El sistema de alojamiento de ambiente controlado no evita la notable variación entre eyaculados dentro y entre los meses evaluados.

\section{Literatura Citada}

1. Argenti LB, Parmeggiani BS, Leiptnitz G, Weber A, Pereira GR, Bustamante IC. 2018. Effect of season on boar semen parameters and antioxidant enzymes in the south subtropical in region in Brazil. Andrologia 50: e12951. doi: 10.1111/and.12951

2. Banaszewska D, Kondracki S. 2012. An assessment of the breeding maturity of insemination boars based on ejaculate quality changes. Folia Biol - Krakow 60: 151-162. doi: 10.3409/fb60_34.151162

3. Cai H, Ren Y, Li XX, Yang JL, Zhang CP, Chen M, et al. 2011. Scrotal heat stress causes a transient alteration in tight junctions and induction of TGF- $\beta$ expression. Int J Androl 34: 352-362. doi: 10.1111/j.1365-2605.2010.01089.x

4. Caisin L, Snitco, T. 2016. The influence of the seasonality on boars sperm production - Breeder of different species. Bulg J Agric Sci 22 (Suppl. 1): 118-122.

5. Dysart NE. 2015. Effect of birth weight and human socialization on reproductive behaviors, sperm production, semen quality, and fertility of AI boars. MSc Thesis. USA: North Carolina State University. $163 \mathrm{p}$.

6. Dziekoñska A, Fraser L, KoziorowskaGilun M, Strzęek J, Koziorowski M, Kordan W. 2014. Seasonal-dependent variations in metabolic status of 
spermatozoa and antioxidant enzyme activity in the reproductive tract fluids of wild boar/domestic pig hybrids. Pol J Vet Sci 17: 307-313. doi: 10.2478/pjvs2014-0042

7. Ewel J, Madriz A, Tosi Jr JA. 1976. Zonas de vida de Venezuela. $2^{\circ}$ ed. Caracas: Ed Sucre. 270 p.

8. Flowers $W .2015$. Factors affecting the efficient production of boar sperm. Reprod Domest Anim 50: 25-30. doi: 10.1111/rda.12529

9. Ford JJ, Wise TH. 2011. Assessment of pubertal development of boars derived from ultrasonographic determination of testicular diameter. Theriogenology 75 : 241-247. doi: 10.1016/j.theriogenology.2010.08.010

10. Fraser L, Strze לek J, Filipowicz, K, Mogielnicka-Brzozowska M, Zasiadczyk L. 2016. Age and seasonaldependent variations in the biochemical composition of boar semen. Theriogenology 86: 806-816. doi: 10.1016/ j.theriogenology.2016.02.035

11. Fuentes $A$, de Serrano $G$, de Manzo M, Regaciro C, Valle A. 1992. Efecto de la época sobre las características espermáticas de verracos en el trópico. Zootec Trop 10 (Suppl.1). [

12. Gómez E. 2016. Valoración del bienestar animal porcino en diferentes condiciones de alojamiento, utilizando indicadores de estrés y parámetros reproductivos. Tesis Doctoral. Madrid: Univ. Complutense de Madrid. $171 \mathrm{p}$.

13. Huang YH, Lo LL, Liu SH, Yang TS. 2010. Age-related changes in semen quality characteristics and expectations of reproductive longevity in Duroc boars. Anim Sci J 81: 432-437. doi: 10.1111/ j.1740-0929.2010.00753.x

14. Knecht D, Erodoñ S, Duziñski K. 2014. The influence of boar breed and season on semen parameters. S Afr J Anim Sci 44: 1-9. doi: 10.4314/ sajas.v44i1.1

15. Knecht D, Srodon S, Szulc K, Duziñski K. 2013. The effect of photoperiod on selected parameters of boar semen.
Livest Sci 157: 364-371. doi: 10.1016/ j.livsci.2013.06.027

16. Koprianiuk A, Kondracki S, Wysokiñska A, Iwanina M, Serewa D. 2014. Season-dependent characteristics of insemination boar ejaculates. Folia Pomer Univ Technol Stetin Agric Aliment Pisc Zootech 15: 31-40.

17. Kunowska-slósarz, M, Makowska A. 2011. Effect of breed and season on the boar's semen characteristics. Ann Warsaw Univ of Life Sci 49: 77-86.

18. Li XX, Chen SR, Shen B, Yang JL, Ji $S Y$, Wen Q, Zheng QS, Li L, Zhang J, Hu ZY, Huang XX, Liu YX. 2013. The Heat-Induced Reversible Change in the Blood-Testis Barrier (BTB) Is Regulated by the Androgen Receptor (AR) via the Partitioning-Defective Protein (Par) polarity complex in the mouse. Biol Reprod 89: 12. doi: 10.1095/biolreprod.113.109405

19. Lipenský J, Lustyková A, Èeoovský J. 2010. Effect of season on boar sperm morphology. J Cent Eur Agric 11: 465468. doi: 10.5513/JCEA01/11.4.866

20. Malmgren, L. 1989. Experimentally induced testicular alternations in boars: sperm morphology changes in mature and peripubertal boars. J Vet Med A 36: 411-420. doi: 10.1111/j.1439-0442.1989.tb00748.x

21. Mažeika K, Sutkeviciene N, Žilinskas H, Riškeviciene V, Aniuliene A, Juodžiukyniene N. 2012. Relationship between sperm quality and testicular lesions in culled AI boars. Vet ZootechLith 59: 52-57.

22. Murphy E, Stanton C, O'Brien C, Murphy C, Holden S, Murphy R, et al. 2017. The effect of dietary supplementation of algae rich in docosahexaenoic acid on boar fertility. Theriogenology 90: 78-87. doi: 10.1016/ j.theriogenology.2016.11.008

23. Olczak K, Nowicki J, Klocek C. 2015. Pig behaviour in relation to weather conditions - a review. Ann Anim Sci 15: 601-610. doi: 10.1515/aoas-2015-0024 
24. Peña P, Stone A, Gummow B, Parker $A$, Paris D. 2019. Tropical summer induces DNA fragmentation in boar spermatozoa: implications for evaluating seasonal infertility. Reprod Fert Develop 31: 590-601. doi.org/10.1071/RD18159

25. Petrocelli H, Batista C, Gosálvez J. 2015. Seasonal variation in sperm characteristics of boars in southern Uruguay. Rev Bras Zootecn 44: 1-7. doi: 10.1590/S1806-92902015000100001

26. Pinart E, Puigmulé M. 2013. Factors affecting boar reproduction, testis function, and sperm quality. In: Bonet $S$, Casas I, Holt W, Yest M (eds). Boar reproduction. Berlin: Springer. p 109-202.

27. Rivera MM, Quintero AA, Barrera $X$, Palomo M, Rigau T, Rodríguez JE. 2005. Natural Mediterranean photoperiod does not affect the main parameters of boar-semen quality analysis. Theriogenology 54: 934-946. doi: 10.1016/j.theriogenology.2005.01.001

28. Savice R, Petroviae M, Radojkovia D, Radovice Ė, Parunovice N. 2013. The effect of breed, boar and season on some properties of sperm. Biotechnol Anim Husb 29: 299-310. doi: 10.2298/ BAH1302299S

29. Saviae R, Petroviae M. 2015. Effect of photoperiod on sexual activity of boar. Rev Bras Zootecn 44: 276-282. doi: 10.1590/S1806-92902015000800002

30. Suriyasomboon A, Lundeheimd N, Kunavongkrite A, Einarsson S. 2004. Effect of temperature and humidity on sperm production in Duroc boars under different housing systems in Thailand. Livest Prod Sci 89: 19-31. doi: 10.1016/ j.livprodsci.2003.12.008

31. Thom EC. 1959. The discomfort index. Weatherwise 12: 57-59. doi: 10.1080/ 00431672.1959 .9926960

32. Wisokiñska A, Kondracki S, Kowalewski D, Adamiak A, Muczyñska E. 2009. Effect of seasonal factors on the ejaculate properties of crossbred Duroc $x$ Pietrain and Pietrain x Duroc boars as well as purebred Duroc and Pietrain boars. Bull Vet Inst Pulawy 53: 677-685.

33. Yeste M, Sancho S, Briz M, Pinart E, Bussalleu E, Bonet S. 2010. A diet supplemented with L-carnitine improves the sperm quality of Pietrain but not of Duroc and Large White boars when photoperiod and temperature increase. Theriogenology 73: 577-586. doi: 10.1016/j.theriogenology.2009.10.013

34. Žaja IZ, Samardžija $M$, Vince $S$, Majice-Balice I, Vilice M, Đurièice D, Milinkovia-Tur S. 2016. Influence of boar breeds or hybrid genetic composition on semen quality and seminal plasma biochemical variables. Anim Reprod Sci 164: 169-176. doi: 10.1016/j.anireprosci.2015.11.027

35. Zasiadczyk L, Fraser L, Kordan W, Wasilewska K. 2015. Individual and seasonal variations in the quality of fractionated boar ejaculates. Theriogenology 83: 1287-1303. doi: 10.1016/ j.theriogenology.2015.01.015 